\title{
The Important Reference Factors of Nuclear Vessel Hydrostatic Test Zhen-Guo ZHANG ${ }^{1, a^{*}}$ \\ ${ }^{1}$ Suzhou Nuclear Power Research Institute Co., Ltd.,Shenzhen,Guangdong, 518124,China a191162990@qq.com
}

Keywords: Nuclear power plant, Vessel, Hydrostatic test

\begin{abstract}
Nuclear vessels hydrostatic test is one of the important test means of the nuclear vessel sealing and pressure resistance, this paper introduces the factors which need to consider when making the nuclear vessel hydrostatic test scheme , (such as: the choice of intake and exhaust point, the determination of isolation boundary, etc.), it provides reference value for nuclear vessel hydrostatic test scheme.
\end{abstract}

\section{Introduction}

The safety of nuclear power plant mainly depends on the function of security equipment reliability, the nuclear pressure vessel plays an irreplaceable role. Most of equipment contains the radioactive medium, once leakage or accident happen, it will cause immeasurable loss and great social influence, so during the equipment service, hydrostatic test is indispensable to ensure the safety of equipment vessel. The main purpose of the hydrostatic test is to examine the vessel pressure resistance and pressure intensity. Nuclear power plant hydrostatic test can be divided into three categories: the primary circuit water pressure test, steam generator secondary side water pressure test and vessel water pressure test, this paper mainly discusses the nuclear vessel hydrostatic test scheme.

The upstream relevant laws and regulations of hydrostatic test mainly includes: the RSE -M PWR nuclear equipment in service inspection rules[1], the RCC -M PWR nuclear machinery and equipment design and construction rules[2]. According to the norms, we can know the basic rules of vessel hydrostatic test, namely the cycle of water pressure test, pressure, examination content, acceptance criteria, etc., with these basic rules, we can refer the EOMR, EOMM, SDM of the vessel and flowcharts to make test program of each vessel. When making nuclear vessel hydrostatic test scheme, reference factors needs to be addressed include: nuclear vessel hydrostatic test range, test pressure, test cycle, the exhaust point selection, the drainage point selection, the determination of isolation boundary, etc.

\section{Nuclear Vessel Hydrostatic Test Scope}

The vessels that require to do hydrostatic test are divided into three categories: steam medium pressure vessel, superheated water pressure vessel, gas medium vessel, the principle of the test pressure is different for different types of vessels category.The classification rules of the three types pressure vessels are as follows:

1) Steam medium pressure vessel must meet the following two conditions at the same time.

$>$ volume is greater than $0.1 \mathrm{~m}^{3}$

$>$ maximum working pressure is greater than $0.5 \mathrm{bar}$

2) Superheated water pressure vessel must meet the following two conditions at the same time.

$>$ maximum working temperature is more than $110^{\circ} \mathrm{C}$

$>$ volume is greater than $0.1 \mathrm{~m}^{3}$

3) Gas medium pressure vessel must meet the following two conditions at the same time.

$>$ operation with the atmospheric pressure is more than $4 \mathrm{bar}$

$>$ pressure (bar) multiplied by the volume (liters) is greater than 80 


\section{Hydrostatic Test Pressure and Inspection Cycle Selection}

\section{Hydrostatic test pressure selection}

The principles are as follows:

1) Steam medium vessel or superheated water pressure vessel test pressure shall not be lower than the table below.

Table 1: Hydrostatic test pressure selection with steam medium vessel or superheated water pressure vessel

\begin{tabular}{|c|c|}
\hline maximum working pressure: $\mathrm{P}$ (bar) & Hydrostatic test pressure: Pt (bar) \\
\hline $0.5<\mathrm{P} \leqslant 2$ & $2 \mathrm{P}$ \\
\hline $2<\mathrm{P}<12$ & $\mathrm{P}+2$ \\
\hline $\mathrm{P} \geq 12$ & $1.17 \mathrm{P}$ \\
\hline
\end{tabular}

2) No gas medium pressure vessel test pressure generally is no less than 1.2 times of the maximum operating pressure, and the test pressure of redo hydrostatic test should be lower than the first hydrostatic test pressure.

3) Gas medium pressure vessel hydrostatic test pressure shall not be lower than 1.5 times of the maximum working pressure.

\section{Inspection Cycle Selection}

According to the request of RSE - M, the hydrostatic test interval of nuclear pressure vessel should not be more than ten years, unless there are special provisions.

\section{The Exhaust Gas, Filling Water, Drainage Point Selection Principle}

\section{Exhaust Gas Point Selection}

The principle is that it generally chooses the top position of the vessel, because it is easy to exhaust gas, and it is better to choose the flange connection or the location of the safety valve or check (the safety valve or check valve will be taken down), because it is easy to install exhaust valve and pressure gauge, it also can reduce the cutting grinding and welding work. Exhaust point number is one commonly, but if there is a long line within the isolation boundary, gas in pipe line is not easy to discharge, it generally needs to design a related exhaust gas point on this position, in order to discharge gas in the long pipe line. If gas in the long pipe line cannot be completely discharge, because the gas has large compressibility, it will influence the speed of test speed.

\section{Filling Water Point Selection}

The principle is that it generally chooses the bottom position of the vessel, and it is better to choose the flange connection or the location of the check valve (the safety valve or check valve will be taken down), because it is easy to connect feed line and test pump, it also can reduce the cutting grinding and welding work. If the job site meets the requirements, the filling water point and drainage points can be set as consistent.

\section{Drainage Point Selection}

The principle is that it generally chooses the bottom position of the vessel, and it is better to choose the flange connection or the location of the pipe cap, because it is easy to connect pipes and reduce cutting grinding and welding work. If it has no similar position, it needs to cut the line at the bottom of the vessel. Drainage pipeline generally choose lowest of the system, but some parts of the pipeline may be twists and turns, sometimes it will leave a little water in the pipe, then the drainage point shall be set up. 


\section{The Determination of Isolation Boundary}

How to determine the isolation boundary of the hydrostatic test is the most critical problem. The probable damaged devices (such as relief valve, expansion joint, etc.) should be dismantled or by blind plate isolation before the test, it needs to verify the isolation boundary components (valves and orifice plate, etc.) can bear the test pressure. In the design of isolation boundary scope, the main consideration is the processing of relevant valves, instrumentation, pipeline within the isolation boundary:

Valve pressure ability can be queried from the valve maintenance manuals. The corresponding processing should be based on the type of valve inside the boundary, different types of valves processing principle are as follows:

The relief valve: guiding type must be blocked or removed; Spring loaded type need to dismantle or blocking, it also can use jacking device screwing in the valve cover to prevent the valve open;

Regulating valve: pneumatic valve should not become the isolation boundary, and it need to keep open;

Manual valve: if it must become the isolation boundary, it must check the consistency of the test pressure and valve pressure rating.

Check valve: it needs to be bypass or split disc, it should not become the isolation boundary, it should be bypass or dismantling its valve core;

Isolation valve: manual valve pressure level must be checked; Pneumatic valve as boundary must check that the pressure drop through the valve due to test pressure is less than or equal to 1.1 times the maximum working pressure drop; Electric valve can be used as a boundary, but in front of the booster to the test pressure, the valve must be closed.

Bellows valves: it shall not be higher than the maximum working pressure;

\section{Other Factors to Consider}

\section{Choice Of Filling Water}

Hydrostatic test of filling water can be divided into two kinds: system water filling and external filling water. Most vessel adopt external filling water way, the advantage is that work staff can control the process of water filling, the disadvantage is that if the vessel volume is larger, the water filling need a long time. System water filling time is shorter, it generally needs the run person to cooperate, only suitable for the voltage regulator discharge pressure tank hydrostatic test at present.

\section{The Scene Space Constraints}

The scene space condition is one of the important factors which needs to be considered to make the hydraulic test. When the scene space is enough, cutting, grinding related pipeline and disassembling TSD facilities are relatively easy to operate, and choosing the exhaust point is also unrestricted. When the scene space is limited, the pipeline cutting, grinding work can't operate, it needs to adjust the position of the related cutting and grinding point, you can find suitable cutting grinding position along the direction of the line to the open space without affecting the test process.

\section{Summary}

As nuclear power units is increasing rapidly in China, the amount of nuclear vessels is also growing exponentially, hydrostatic test work importance is becoming more and more significant. The implementation of water pressure test, can not only test the nuclear vessel pressure resistance and the sealing performance, but also can shorten the time of the plant overhaul and create huge economic benefits. This paper discusses the key factors which need to consider when making the nuclear vessel hydrostatic test scheme, it has a certain reference significance for the engineering and technical personnel who need to implement the hydrostatic test work .

\section{Reference}


[1] RSE-M In-service Inspection Rules for the Mechanical Components of PWR Nuclear Island, AFCEN, 1997 edition.

[2] RCC-M Design and Construction Rules for Mechanical Components of PWR Nuclear Islands, AFCEN, 2007 edition. 